\title{
The experience of breast reconstructive microsurgery
}

\author{
Bayu Brahma1,2, Samuel J Haryono ${ }^{1,3}$ \\ ${ }^{1}$ Department of Surgical Oncology, Dharmais Hospital, ${ }^{2}$ Department of Surgical \\ Oncology, Kota Bogor General Hospital, ${ }^{3}$ Department of Surgical Oncology, \\ MRCCC Siloam Hospital
}

DOI: http://dx.doi.org/10.19106/JMedScieSup004804201603

\section{ABSTRACT}

Autologous techniques in oncoplastic breast surgery might result in graft donor site morbidity. Microsurgery has become a new surgical modality for breast reconstruction, as it is less invasive. In recent experience, we have applied microsurgical technique in oncoplastic breast procedures to minimize morbidity. We reviewed the charts of breast cancer/tumor patients treated with microsurgical reconstruction. From February 2013 to July 2016, we performed 36 perforator flaps for breast reconstruction. The mean age of the patients was $44.4 \pm 6.7$ years old, with the median tumor size of $3.7(1.5-20) \mathrm{cm}$. No special type of carcinoma (NST) was accounted in $25(69.4 \%)$ cases. Oncoplastic breast conserving surgery (OPS) was the procedure of choice in 17 (47.2\%) cases and mastectomy was followed by free flap in $19(52.8 \%)$ patients. In OPS, we used various perforator flaps to cover the defect. Thoracodorsal artery perforator flap (TDAP) was the most common technique used in $8(22.2 \%)$ cases, lateral intercostal artery (LICAP) flap in $6(16.7 \%)$ cases, anterior intercostal artery (AICAP) flap in $1(2.8 \%)$ cases, and superficial epigastric artery (SEAP) flap in $2(5.6 \%)$ cases. Deep inferior artery perforator (DIEP) free flap was the option for reconstruction after mastectomy. During follow-up (mean time of $12.7 \pm 11.4$ months), there were 1 local recurrence, 2 regional and systemic metastases, and 1 death due to cerebrovascular disease. There was no flap loss after pedicle perforator reconstruction, yet total flap necrosis occurred in 5 patients with DIEP free flap. In one patient, we successfully salvaged the flap with venous congestion. There was no seroma at donor site and no limitation in abdominal wall function after DIEP reconstruction. In conclusion, microsurgical reconstruction in breast surgery has been a safe procedure with less donor site morbidity. Flap failure rate might be improved by refining microsurgical technique.

Keywords: breast cancer; microsurgical reconstruction; oncoplastic 